jnnp-2016-314600 (letter)

INTRASPINAL PRESSURE AND SPINAL CORD PERFUSION PRESSURE

PREDICT NEUROLOGICAL OUTCOME AFTER TRAUMATIC SPINAL CORD

INJURY

Samira SAADOUN, Suliang CHEN, Marios C. PAPADOPOULOS

Academic Neurosurgery Unit, St. George's, University of London, London SW17 0RE, U.K.

Correspondence to: Prof. Marios Papadopoulos, Department of Neurosurgery, St. George's Hospital, London SW17 0QT. Email.mpapadop@sgl.ac.uk, Tel. +44-20-87254179, Fax. +44-20-87254452.

Word count: Text 998 


\section{INTRODUCTION}

In the U.K., blood pressure management after acute, severe traumatic spinal cord injury (TSCI) is variable [1]. The American Association of Neurological Surgeons (AANS) recommends maintaining mean arterial pressure (MAP) at $85-95 \mathrm{mmHg}$ for a week after injury, but with little supporting evidence [2]. To rationalise blood pressure management, we monitor intraspinal pressure (ISP) and spinal cord perfusion pressure (SCPP=MAP-ISP) from the injury site [3]. Our technique is safe [4] and analogous to monitoring intracranial pressure (ICP) and cerebral perfusion pressure (CPP) in traumatic brain injury (TBI). The data indicate that, after TSCI, ISP rises and SCPP falls [3]. Here we show strong correlation between high ISP or low SCPP and reduced neurological recovery. Our findings raise the possibility that interventions to reduce ISP or increase SCPP after TSCI may improve neurological outcome.

\section{METHODS}

Patient recruitment Approval for this observational cohort study was granted by the National Research Ethics Service London-St Giles Committee. Inclusion criteria are: 1. Severe TSCI defined as American spinal injuries association Impairment Scale (AIS) grade A, B or C; 2. Age 18-70 years; 3. Timing between TSCI and surgery within 72 hours. Exclusion criteria are: 1. Patient unable to consent; 2. Other major injuries or co-morbidities; 3 Penetrating TSCI. Surgery and early management took place at St. George's Hospital. Consent was obtained by each patient.

Surgery Following bony realignment and posterior fixation, the ISP probe (Codman, Depuy Synthes, Leeds, UK) was tunnelled through skin into the wound cavity. Under the operating microscope, the dura was opened one level below the injury. The ISP probe was inserted through the durotomy and placed on the spinal cord surface (Fig. 1A). The dural opening was 
sutured and supplemented with fibrin glue (Tisseel ${ }^{\circledR}$, Baxter, UK). The ISP probe was secured to the skin with silk sutures. Details are given elsewhere [3].

ISP and SCPP monitoring The ISP probe was connected to a Codman ICP box linked via a ML221 amplifier to a PowerLab running LabChart v.7.3.5 (AD Instruments, Oxford, UK). Arterial blood pressure was recorded from a radial artery catheter connected to the Philips Intellivue MX800 bedside monitoring system (Philips, Guildford, UK) in turn connected to the PowerLab system. The ISP and arterial blood pressure signals were sampled for up to seven days. LabChart was used to analyse the signals and compute SCPP. Neurointensivists were blinded to the ISP reading. MAP was treated with noradrenaline according to the neurointensivists' preferences.

Data collection Neurological examinations were done pre-operatively (day of injury) and at 9-12 months. Whole spine CT and MRI were done on admission and another CT within 48 hours of surgery to check probe and screw position. We collected the following: patient age, AIS grade pre-operatively and at 9-12 months, level of injury, ISP and SCPP.

Statistics Data were analysed using exact asymptotic logistic regression (LogXact 11, Cytel, Cambridge MA, USA). Neurological outcome was AIS grade at follow-up vs. pre-operative, binarised as improved $v s$. non-improved. We first assessed each factor individually. A forward stepwise method was then used to construct multi-variate models.

\section{RESULTS}

There were 45 patients with average age 41 years (range 19-70) and male:female ratio 4:1. 53\% patients had cervical (C2-C7), 22\% upper thoracic (T1-T6), 7\% lower thoracic (T7T10) and 18\% conus medullaris (T11-L2) injuries. Pre-operative AIS grade was A in 67\% patients, B in $20 \%$ and $\mathrm{C}$ in 13\%. For details see Supplementary Table 1. In univariate analysis, AIS grade on admission $(\mathrm{P}<0.05)$, advanced patient age $(\mathrm{P}<0.005)$ and upper 
thoracic level of injury $(\mathrm{P}<0.05)$ correlated with reduced chance of AIS improvement by at least one grade at 9-12 months. Patient sex, duroplasty or laminectomy did not predict neurological outcome. For each patient, we computed mean ISP, mean SCPP and mean MAP during the monitoring period. In univariate analysis, ISP (negative correlation, $\mathrm{P}<0.05$ ) and SCPP (positive correlation, $\mathrm{P}<0.05$ ), but not MAP correlated with AIS grade improvement at 9-12 months (Figs. 1B-D). For details see Supplementary Table 2.

Multivariate analysis was used to determine whether AIS grade on admission, injury level, patient age, mean ISP and mean SCPP are independent predictors of improvement by at least one AIS grade. We produced two models. In one model, age, AIS grade on admission and ISP were independent predictors of AIS improvement $(\mathrm{P}<0.05)$. Increase in ISP by 10 mmHg reduced the chance of AIS grade improvement about five times. In the other model, age, AIS grade on admission and SCPP were independent predictors of AIS improvement $(\mathrm{P}<0.05)$. Increase in SCPP by $10 \mathrm{mmHg}$ increased the chance of AIS grade improvement about 2.7 times. Due to collinearity, ISP and SCPP were not predictors in the same model. For details see Supplementary Table 3.

\section{DISCUSSION}

Our key finding is that injury site ISP and SCPP, monitored during the first few days after TSCI, predict neurological improvement at 9-12 months. Mean ISP $<10 \mathrm{mmHg}$ and mean SCPP $>90 \mathrm{mmHg}$ are associated with the best recovery. In multivariate analysis, elevated ISP or low SCPP is a significant risk factor independent of AIS grade on admission and patient age. Limitations of the study include the small number of patients and use of the AIS as the only outcome measure.

Our findings for TSCI are analogous to the corresponding relationships between ICP, CPP and recovery after TBI. Spinal cord microdialysis indicates that high ISP and low SCPP 
are associated with injury site ischaemia [5], which likely causes secondary damage. Because only one patient had mean SCPP $>100 \mathrm{mmHg}$, it is impossible to determine what happens to neurological outcome when mean SCPP is $>90-100 \mathrm{mmHg}$. There was no correlation between the AANS MAP guideline of $85-95 \mathrm{mmHg}$ and recovery. In our view, MAPtargeted management does not make physiological sense because patients with the same MAP have different SCPP; our patients with MAP 85-95 mmHg had SCPP 50-90 mmHg. Ultimately, a randomised, controlled trial is required to determine whether interventions that reduce ISP or increase SCPP improve neurological outcome after TSCI.

\section{ACKNOWLEDGMENTS}

Funded by Wings for Life Spinal Cord Research Foundation and Neurosciences Research Foundation (Fletcher fund). We thank the neurosurgery departments at St. George's Hospital, King's College Hospital and Hurstwood Park Neurological Centre for help with patient recruitment. We also thank the anaesthetic, neuro-intensive care unit and operating theatre staff at St. George's Hospital for help with catheter insertion and data collection.

\section{REFERENCES}

1. Werndle MC, Zoumprouli A, Sedgwick P, et al. Variability in the treatment of acute spinal cord injury in the United Kingdom: results of a national survey. J Neurotrauma 2012;29:8808.

2. Cozzens JW, Prall JA and Holly L. The 2012 Guidelines for the Management of Acute Cervical Spine and Spinal Cord Injury. Neurosurgery 2013;72 Suppl 2:2-3.

3. Werndle MC, Saadoun S, Phang I, et al. Monitoring of spinal cord perfusion pressure in acute spinal cord injury: initial findings of the injured spinal cord pressure evaluation study. Crit Care Med 2014;42:646-55. 
4. Phang I, Zoumprouli A, Saadoun S, et al. Safety profile and probe placement accuracy of intraspinal pressure monitoring for traumatic spinal cord injury: Injured Spinal Cord Pressure Evaluation study. J Neurosurg Spine 2016;25:398-405.

5. Phang I, Zoumprouli A, Papadopoulos MC, et al. Microdialysis to Optimize Cord Perfusion and Drug Delivery in Spinal Cord Injury. Ann Neurol 2016;80:522-31. 


\section{FIGURE LEGEND}

Fig. 1. Relationship between monitored physiological parameters and neurological

outcome. A. Schematic of ISP monitoring technique. \% patients with AIS grade

improvement at 9 - 12 months $v s$. B. mean ISP, C. mean SCPP, and D. mean MAP. Black

bars show improvement by at least one AIS grade. White bars show improvement by at least

2 AIS grades. $85-95 \mathrm{mmHg}$ MAP is the AANS recommendation. 
A

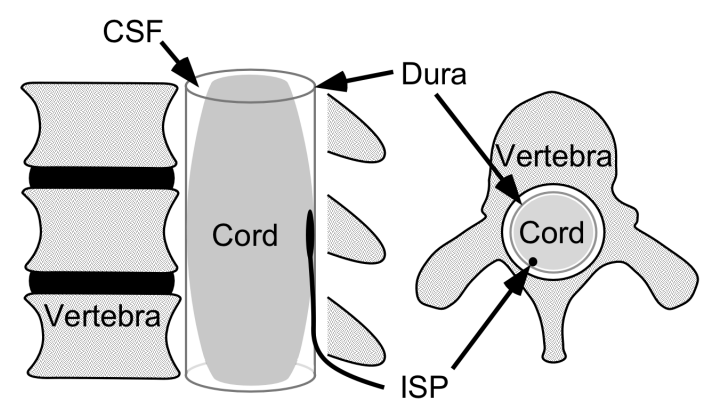

C Improvement (\% of patients)

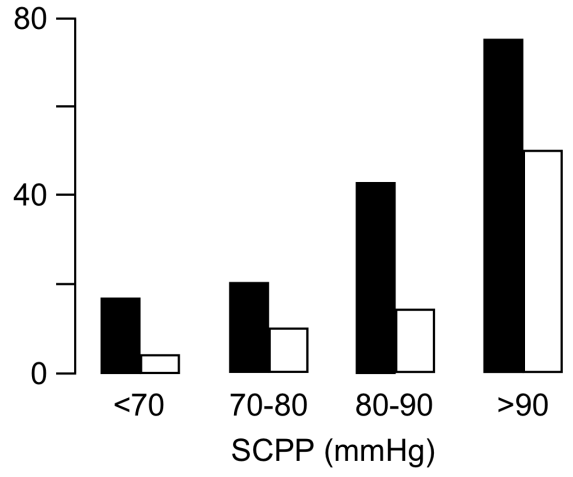

B

Improvement (\% of patients)

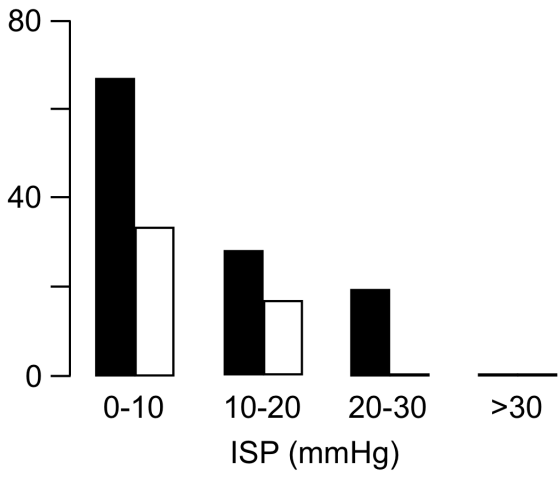

D Improvement (\% of patients)
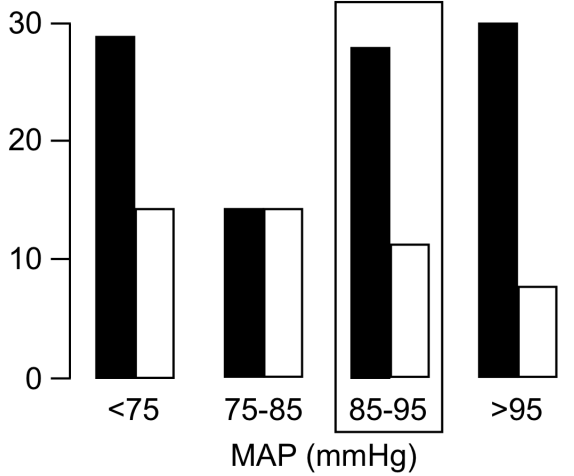

Figure 1 


\section{INTRASPINAL PRESSURE AND SPINAL CORD PERFUSION PRESSURE}

\section{PREDICT NEUROLOGICAL OUTCOME AFTER TRAUMATIC SPINAL CORD}

\section{INJURY}

Samira SAADOUN, Suliang CHEN, Marios C. PAPADOPOULOS

SUPPL Table 1. Demographic details of patients included in this study.

\begin{tabular}{|l|l|}
\hline AGE, mean (range) & 41 (19-70) \\
\hline SEX, m:f patients & $36: 9$ \\
\hline ADMISSION AIS GRADE, no. of patients & $30 \mathrm{~A}, 9 \mathrm{~B}, 6 \mathrm{C}$ \\
\hline FOLLOW-UP AIS GRADE, no. of patients & $26 \mathrm{~A}, 6 \mathrm{~B}, 3 \mathrm{C}, 8 \mathrm{D}, 1 \mathrm{E}, 1$ ? \\
\hline INJURY LEVEL, no. of patients & $24 \mathrm{C} 2-7,10 \mathrm{T1}-6,3 \mathrm{~T} 7-10,8 \mathrm{~T} 11-\mathrm{L} 2$ \\
\hline *EXTENT OF DECOMPRESSION, no. of patients & $11 \mathrm{R}, 24 \mathrm{R} / \mathrm{L}, 10 \mathrm{R} / \mathrm{L} / \mathrm{D}$ \\
\hline TIME INJURY TO SURGERY, mean (range) & 37 hours (9-72) \\
\hline
\end{tabular}

${ }^{*}$ All patients had posterior surgery. For cervical spine, we used lateral mass screws with rods and for thoracolumbar spine pedicle screws with rods. We restored the normal alignment of the fractured spine (R). Some patients also had laminectomies spanning the injury $(R / L)$ and some had laminectomy plus duroplasty $(R / L / D)$. 
INTRASPINAL PRESSURE AND SPINAL CORD PERFUSION PRESSURE

PREDICT NEUROLOGICAL OUTCOME AFTER TRAUMATIC SPINAL CORD

INJURY

Samira SAADOUN, Suliang CHEN, Marios C. PAPADOPOULOS

SUPPL Table 2. Factors tested in univariate analysis. Outcome was improvement in AIS grade at 9-12 months compared with AIS grade on admission.

\begin{tabular}{|l|l|}
\hline \multicolumn{1}{|c|}{ FACTOR } & P $<\mathbf{0 . 0 5 ?}$ \\
\hline Age & YES \\
\hline Sex & NO \\
\hline Level of injury & YES \\
\hline Extent of decompression (R, R/L, R/L/D) & NO \\
\hline AIS grade on admission & YES \\
\hline Mean ISP & YES \\
\hline Mean SCPP & YES \\
\hline Mean MAP & NO \\
\hline
\end{tabular}




\title{
INTRASPINAL PRESSURE AND SPINAL CORD PERFUSION PRESSURE PREDICT NEUROLOGICAL OUTCOME AFTER TRAUMATIC SPINAL CORD INJURY
}

\author{
Samira SAADOUN, Suliang CHEN, Marios C. PAPADOPOULOS
}

SUPPL Table 3. Details of the two exact logistic regression models, using AIS improvement by at least one grade at $9-12$ months as neurological outcome. We first listed the predictors that were statistically significant in univariate analysis at $\mathrm{P}<0.05$ i.e. patient age, AIS on admission, level of injury, ISP and SCPP. Though commonly $\mathrm{P}<0.25$ is used, for each of our predictors $\mathrm{P}$ was either $<0.05$ or $>0.25$. To produce the multi-variate model, we started with no predictors and proceeded forward according to the following algorithm: 1) Add to the multivariate model the predictor with the lowest P-value (from univariate analysis). 2) Recalculate P-values for each predictor in multivariate model. 3) Remove predictor from multivariate model with $\mathrm{P}>0.05$. 4) Stop if adding a predictor to multivariate model causes $\mathrm{P}$ value of that predictor to $>0.05$.

\begin{tabular}{|c|c|c|c|c|}
\hline PREDICTOR & P-VALUE & BETA & BETA SE & ODDS RATIO \\
\hline \multicolumn{5}{|l|}{${ }^{\dagger}$ MODEL 1} \\
\hline *Age & 0.006 & -1.179 & 0.495 & 3.3 \\
\hline Admission AIS grade & 0.015 & 1.156 & 0.678 & 4.8 \\
\hline${ }^{\#}$ ISP & 0.016 & -1.608 & 0.727 & 5.0 \\
\hline \multicolumn{5}{|l|}{${ }^{\S}$ MODEL 2} \\
\hline *Age & 0.004 & -1.225 & 0.499 & 3.4 \\
\hline Admission AIS grade & 0.027 & 1.446 & 0.654 & 4.3 \\
\hline${ }^{\#} \mathrm{SCPP}$ & 0.026 & 0.995 & 0.454 & 2.7 \\
\hline
\end{tabular}

